\title{
Letters
}

\section{Traumatic spinal cord injuries in Istanbul}

I read with interest 'Traumatic spinal cord injuries in Istanbul, Turkey. An Epidemiological study' (Karamehmetoglu et al, Paraplegia 1995; 33: 469-471).

However I was surprised both by the absence of any reference to sports injury and by the absence of the inclusion in the list of references of their Ankaran compatriots' paper 'Wrestling causing paraplegia' (Acikgos et al, Paraplegia 1990; 28: 265-268).

Should we conclude that Turkish wrestling is restricted to central and eastern Turkey or have there been advances in the safety of Turkish wrestling in recent years?

Mr RG Pringle FRCS Consultant Orthopaedic Surgeon Burnell House 82 Berwick Road Shrewsbury, England

\section{Reply from Dr Safak S Karamehmetoglu}

The paper 'Wrestling causing paraplegia' (B Acikgoz et al, Paraplegia 1990; 28: 265-268) was a case presentation where four cases were discussed. The first patient had a $\mathrm{C} 5-6$ right posterolateral intervertebral disc herniation with diminished deep tendon reflexes. As far as I know, he cannot be accepted as having a spinal cord injury (SCI) by definition (Standards for Neurological and Functional Classification of Spinal Cord Injury, Revised 1992, ASIA, American Spinal Injury Association, p.5).

The second patient, with an anterior dislocation of $\mathrm{C} 3$ on $\mathrm{C} 4$ was quite normal on neurological examination. There was a spinal vertebral column injury but no spinal cord damage. The third patient was tetraplegic due to a C5-6 fracture-dislocation. He was injured whilst wrestling with a friend but not in an official match.

The fourth patient was thrown onto his neck during an official wrestling match. He had a C3-4 dislocation causing tetraplegia. It was not the Traditional Turkish Wrestling (TTW) because you cannot throw the wrestler in TTW as the wrestlers use olive oil to make their bodies slippery.

These four patients were reported from the Hacettepe University Medical Faculty, Neurosurgery department between 1968-1983 (two tetraplegics due to wrestling in 16 years), thus the incidence of spinal paralysis was very low and neither was due to TTW.

In our epidemiological study, we reported all new instances of SCI in one year and only one was due to a sports accident (soccer).

As far as I know, there are no changes in the rules of TTW and it is very unlikely that SCI will result from TTW which has been practiced since ancient times all over our country.

Dr Safak S Karamehmetoglu Kizilelma cad Omer Seyfettin Findikzade Istanbul, Turkey

\section{Plaque and gingivitis in spinal paralysed individuals}

Toothbrushing with a toothpaste is arguably the most common oral hygiene method of developed nations. ${ }^{1}$ Practiced efficiently, toothcleaning will maintain satisfactory gingival health ${ }^{2}$ and if a fluoride toothpaste is used, reduce caries experience. ${ }^{3}$ Any physical and/or mental handicap invariably influences toilet and grooming habits, including tooth cleaning. Indeed in handicapped individuals it is not unusual for oral hygiene to be delegated to a parent, guardian or other care worker. Depending upon the severity of any handicap overall hygiene care tends to be prioritized and may be influenced by the experience, ability and available time of the carer. What is certain is failure to ensure satisfactory oral hygiene will always result in chronic gingivitis, ${ }^{2}$ with the possibility in time for progression to chronic periodontitis.

Studies of handicapped individuals have usually revealed gingival and dental health problems with significantly poorer oral hygiene compared to the non handicapped. ${ }^{5,6}$ For this reason it has been recommended that patients, parents and caring staff require from an early stage, dental health education and active involvement in prevention programmes.?

Where severe physical handicap occurs, the mouth takes on a significant role with the oral/facial and masticatory muscles being the few skeletal muscles systems which still function, to this end alone, the maintenance of a healthy mouth would seem most important.

A study undertaken by the authors in 1994 assessed the oral hygiene and gingival health status of paraplegic in patients at a specialised rehabilitation centre in Switzerland. Plaque indices (Silness and Loe) ${ }^{8}$ and the presence or absence of gingival bleeding sites were noted for a group of 24 patients (12 paraplegic and 12 tetraplegic), in a small cross sectional needs assessment survey. Although it was apparent that some individuals had good oral hygiene, only three had plaque scores less than 1 for the index (range $0-3$ ). The remainder had plaque scores of greater than 1 and extending to 2.5 , which would place more than $85 \%$ of patients in the moderate to poor oral hygiene category. A strong correlation between plaque and gingivitis was highly significant, a strong association noted in large epidemiological studies. ${ }^{4,9}$ Almost $50 \%$ average bleeding sites emphasised the gingival problems of the group.

The pattern was for poor oral hygiene and gingival health in the tetraplegic patients and the difference between the two groups is significant. Given the extent of the handicap, these findings would be expected and emphasise the dependant nature of those who are tetraplegic for all hygiene procedures including tooth cleaning.

Given the long term consequences of poor oral hygiene and gingival health in a handicapped population where oral function is vital, there would appear to be a need to institute preventive programmes. Such programmes may be provided for and performed by the individual or delegated to third parties depending upon the degree of handicap. Chemical adjuncts such as fluoride and chlorhexidine products may be considered. 